\title{
The Arecibo Galaxy Environment Survey - X. The structure of halo gas around M33
}

\author{
O. C. Keenan, ${ }^{1 \star}$ J. I. Davies, ${ }^{1}$ R. Taylor ${ }^{2}$ and R. F. Minchin ${ }^{3}$ \\ ${ }^{1}$ School of Physics and Astronomy, Cardiff University, Queens Buildings, The Parade, Cardiff CF24 3AA, UK \\ ${ }^{2}$ Astronomical Institute of the ASCR, Boční II 1401, CZ-14100 Prague, Czech Republic \\ ${ }^{3}$ Arecibo Observatory, HCO3 Box 53995, Arecibo 00612, Puerto Rico
}

Accepted 2015 November 13. Received 2015 November 10; in original form 2015 February 13

\begin{abstract}
As part of the H I Arecibo Galaxy Environment Survey (AGES), we have observed $5 \times 4 \mathrm{deg}$ of sky centred on M33, reaching a limiting column density of $\sim 1.5 \times 10^{17} \mathrm{~cm}^{-2}$ (line width of $10 \mathrm{~km} \mathrm{~s}^{-1}$ and resolution $3.5 \mathrm{arcmin}$ ). We particularly investigate the absence of optically detected dwarf galaxies around M33, something that is contrary to galaxy formation models. We identify 22 discrete H I clouds, 11 of which are new detections. The number of objects detected and their internal velocity dispersion distribution is consistent with expectations from standard galaxy formation models. However, the issue remains open as to whether the observed velocity dispersions can be used as a measure of the H I clouds total mass, i.e. are the velocities indicative of virialized structures or have they been influenced by tidal interactions with other structures in the Local Group? We identify one particularly interesting H I cloud, AGESM33-31, that has many of the characteristics of $\mathrm{H}$ I distributed in the disc of a galaxy, yet there is no known optical counterpart associated with it. This object has a total H I mass of $1.22 \times 10^{7} \mathrm{M}_{\odot}$ and a diameter of $18 \mathrm{kpc}$ if at the distance of $\mathrm{M} 33\left(D_{\mathrm{M} 33}=840 \mathrm{kpc}\right)$. However, we also find that there are numerous other $\mathrm{H}$ I clouds in this region of sky that have very similar velocities and so it is plausible that all these clouds are actually associated with debris from the Magellanic stream.
\end{abstract}

Key words: galaxies: clusters: general-galaxies: dwarf-Local Group-radio lines: galaxies.

\section{INTRODUCTION}

M33 is the third most massive galaxy in the Local Group [after Andromeda (M31) and the Milky Way]. It is generally thought that M31 and M33 are closely associated with each other, for example they may have gravitationally interacted with each other in the relatively recent past (e.g. Bekki 2008). The M31-33 system collectively has around 30 known satellite (dwarf galaxy) companions (Martin et al. 2009), almost all of which are confirmed satellites of M31 with no obvious association with M33. However, Martin et al. (2009) recently announced the discovery of two new dwarf galaxies in close proximity to both M31 and M33. One of these they classified as a definite M31 satellite, but the other, Andromeda XXII, is the first proposed satellite of M33 (e.g.Chapman et al. 2013). And XXII is a lot closer in projection to M33 ( 40 kpc) than to M31 ( 220 kpc) and lies in a region where M33's dark matter halo would appear to dominate gravitationally over that of M31's. The two newly discovered dwarf galaxies were found using the
Pan-Andromeda Archaeological Survey (PAndAS CUBS) optical survey by searching for spatial over densities of stars. In fact almost all previous surveys for faint Local Group dwarf galaxies have relied upon optical searches for these stellar over densities. Given the very low optical surface brightness of the objects detected there may come a point where observations at other wavelengths, like those described here, may become even more productive at finding dwarf galaxies.

With specific regard to M33, extended star clusters have also been found, which Chapman et al. (2013) propose may be tidally stripped dwarf galaxies because of their structure - they are extremely sparse star clusters with elongated distributions of stars. There are also other detections of objects in the halo and around M33. Huxor et al. (2009) show that the globular cluster population of M33 is weighted towards the far side of M33 with respect to M31, which they cite as further evidence of an interaction with M31 having affected M33's halo population. Sarajedini et al. (2006) have identified RR Lyrae stars in the halo of M33. RR Lyrae stars are found in stellar populations that are older than $10 \mathrm{Gyr}$. This helps to determine the age of the halo population of M33 and therefore place a limit on when an interaction with M31 may have occurred. As we will see 
below these stars are considerably older than derived ages for an M31/M33 interaction suggesting that M33's halo may well have been rather unperturbed over quite long time-scales.

Given the proximity of M33 (we will use a distance of $840 \mathrm{kpc}$ throughout this paper Freedman, Wilson \& Madore 1991) and the past optical searches for stars, e.g. Martin et al. (2009), it is surprising that currently there is only one known possible dwarf galaxy companion of M33. It is even more surprising given the standard galaxy formation models that predict many hundreds of dwarf satellite galaxies surrounding larger galaxies like M33 (Stadel et al. 2009 and references therein). So, do the dwarf companions exist, but are still not detected by optical surveys (maybe because of their extremely low surface brightness), or have the dwarfs been destroyed by an M33/M31 interaction? In this paper, we will investigate the halo of M33 not by observing the emission from stars, but by using the $21 \mathrm{~cm}$ emission from atomic hydrogen. Atomic hydrogen is the fuel for dwarf galaxy (star) formation, and is also easily drawn out of galaxies when they gravitationally interact, and as such should offer us some insight into the current nature of M33's halo.

There has been considerable previous work, utilizing $21 \mathrm{~cm}$ observations, that has been used to investigate the halo of M33 and the nature of its relationship with M31. (Braun \& Thilker 2004, hereafter BT04) used the Westerbork array to detect H I over a $1800 \mathrm{deg}^{2}$ region of the sky that covered both M31 and M33. Their survey had a very low $\mathrm{H}_{\mathrm{I}}$ column density sensitivity of $1.5 \times 10^{17} \mathrm{~cm}^{-2}$ over a $30 \mathrm{~km} \mathrm{~s}^{-1}$ velocity width, but a rather large spatial resolution of 49 arcmin (FWHM, full width at half-maximum). They detected $95 \mathrm{H}$ i clouds almost all of which seem to be clearly associated with M31. They also identified a faint $\mathrm{H}$ I stream seemingly joining M33 and M31 however, they were unable to trace this all the way to M33 due to confusion with $\mathrm{H}_{\mathrm{I}}$ in the outskirts of the Milky Way. They detected one $\mathrm{H}_{\mathrm{I}}$ cloud in the region of $\mathrm{M} 33$ with an $\mathrm{HI}$ mass of a few times $10^{5} \mathrm{M}_{\odot}$ - this cloud has properties similar to the high-velocity clouds (HVCs) found around the Milky Way. HVCs are clouds of $\mathrm{H}_{\mathrm{I}}$ with velocities than cannot be explained with a simple model of galactic rotation - Galactic HVCs typically have velocities which deviate from expectations of rotation by $>50 \mathrm{~km} \mathrm{~s}^{-1}$ (Wakker 1991). Westmeier, Braun \& Thilker (2005) present high-resolution ( $\sim 2$ arcmin) follow-up observations of the previously identified M31/M33 H I clouds and again conclude that the single M33 HVC displays very similar characteristics to Galactic HVCs. A possible link between HVCs and the missing dwarf galaxy population as either progenitors of, or failed, dwarf galaxies has previously been proposed by Blitz et al. (1999, 2004).

BT04 propose two possible mechanisms for the origin of the $\mathrm{H}_{\mathrm{I}}$ stream between M31 and M33: (1) that it is a pre-existing filament extending between the two galaxies, filaments like this are a prediction arising from high-resolution numerical models of structure formation (Davé et al. 1999, 2001) or (2) the stream has arisen from a past tidal interaction between M31 and M33. Using the measured systemic velocities of M33 and M31 BT04 concluded that the two galaxies are currently moving towards each other, indicating that no recent ( $<2$ Gyr) interaction has taken place. Putman et al. (2009) have used similar methods to BT04 (an assessment of possible orbits given the observed velocities) and conclude that the galaxies are likely to have interacted between 1 and $3 \mathrm{Gyr}$ ago, which is roughly consistent with BT04. Putman et al. (2009) list distinct features of the gaseous disc of M33 including: an extended warp in the disc, a filament from the northern part of the disc, diffuse gas surrounding the galaxy, and a cloud with a filament extending from the galaxy. They say that all of these features strongly indicate a past interaction between the two galaxies and that this interaction is the probable origin of the M31-M33 stream. Bekki (2008) have carried out an ensemble of simulations of possible orbits for M33 and M31 and conclude that an interaction occurred even longer ago than proposed above - between 4 and $8 \mathrm{Gyr}$.

However, using observations of the stream down to an almost identical BT04 column density limit of $2.7 \times 10^{17} \mathrm{~cm}^{-2}$ for a line width of $25 \mathrm{~km} \mathrm{~s}^{-1}$ Wolfe et al. (2013) report that about 50 per cent of the atomic hydrogen in the stream is composed of distinct clouds whilst what remains is made up of an extended, diffuse component. They are able to identify these distinct clouds because of their much improved spatial resolution of 9 arcmin compared to that of BT04 (49 arcmin). Wolfe et al. (2013) propose an intergalactic filamentary origin of the stream. Basically they argue that the collapse time-scale for the individual clouds they identify is much shorter ( $\sim 400 \mathrm{Myr})$ than the likely time since an interaction (a few Gyr). Wolfe et al. (2013) propose that the clouds may have several possible origins: (1) they may be primordial gas-rich objects similar to dwarf spheroidal or dwarf irregular galaxies, (2) the clouds may be gas accreting into subhaloes, (3) the clouds may be tidal dwarf galaxies in formation and (4) the clouds may be transient objects condensing from a pre-existing intergalactic filament.

Clearly, there is still some lack of agreement as to the origin of the $\mathrm{H}$ I stream/filament. If it is tidal then at first sight it is difficult to see how distinct clouds along its length will not have already collapsed into much smaller denser structures. However, this is based on the assumption that the observed velocity widths measure the 'local' gravitational field and are not highly biased by streaming motions along the filament - something that cannot be ruled out. The question also remains open as to whether any of the $\mathrm{H}$ I condensations could eventually become dwarf galaxies and so help explain the discrepancy between the numbers that theory predicts and the observations.

In this paper, we intend to explore these issues further, with a particular emphasis on whether any of the complex Hi structures seen around and in the halo of M33 could be part of a significant population of M33 dwarf galaxies, and so account for the lack of optically detected companions.

The data described in this paper extends both the column density limit and spatial resolution, of the surveys described above - we reach $\sim 1.5 \times 10^{17} \mathrm{~cm}^{-2}$ for a line width of $10 \mathrm{~km} \mathrm{~s}^{-1}$ with a spatial resolution 3.5 arcmin. Our survey also extends that of (Grossi et al. 2008, hereafter G08) who used the Arecibo Legacy Fast ALFA (ALFALFA) survey observations of a differently shaped area to us around M33 and with lower sensitivity (see below). Our deeper Arecibo observations form part of the Arecibo Galaxy Environment Survey (AGES). The survey is fully described in Auld et al. (2006).

In the following sections, we will describe the observational data and its reduction (Section 2), our method of source identification and measurement (Section 3), an analysis of our results (Section 4), a discussion putting our results in context with regard to the astrophysical problems discussed above (Section 5) and finally a summary of our work and conclusions (Section 6).

\section{OBSERVATIONS AND DATA REDUCTION}

The observations and data reduction techniques are described in depth in Auld et al. (2006), and also in Cortese et al. (2008), Minchin et al. (2010) and Davies et al. (2011). Therefore, they have only been summarized here. Observations of this field began in 2008 August and were completed in 2013 August. The field observed to full depth spans approximately $5 \mathrm{deg}$ of RA by $4 \mathrm{deg}$ of declination centred 


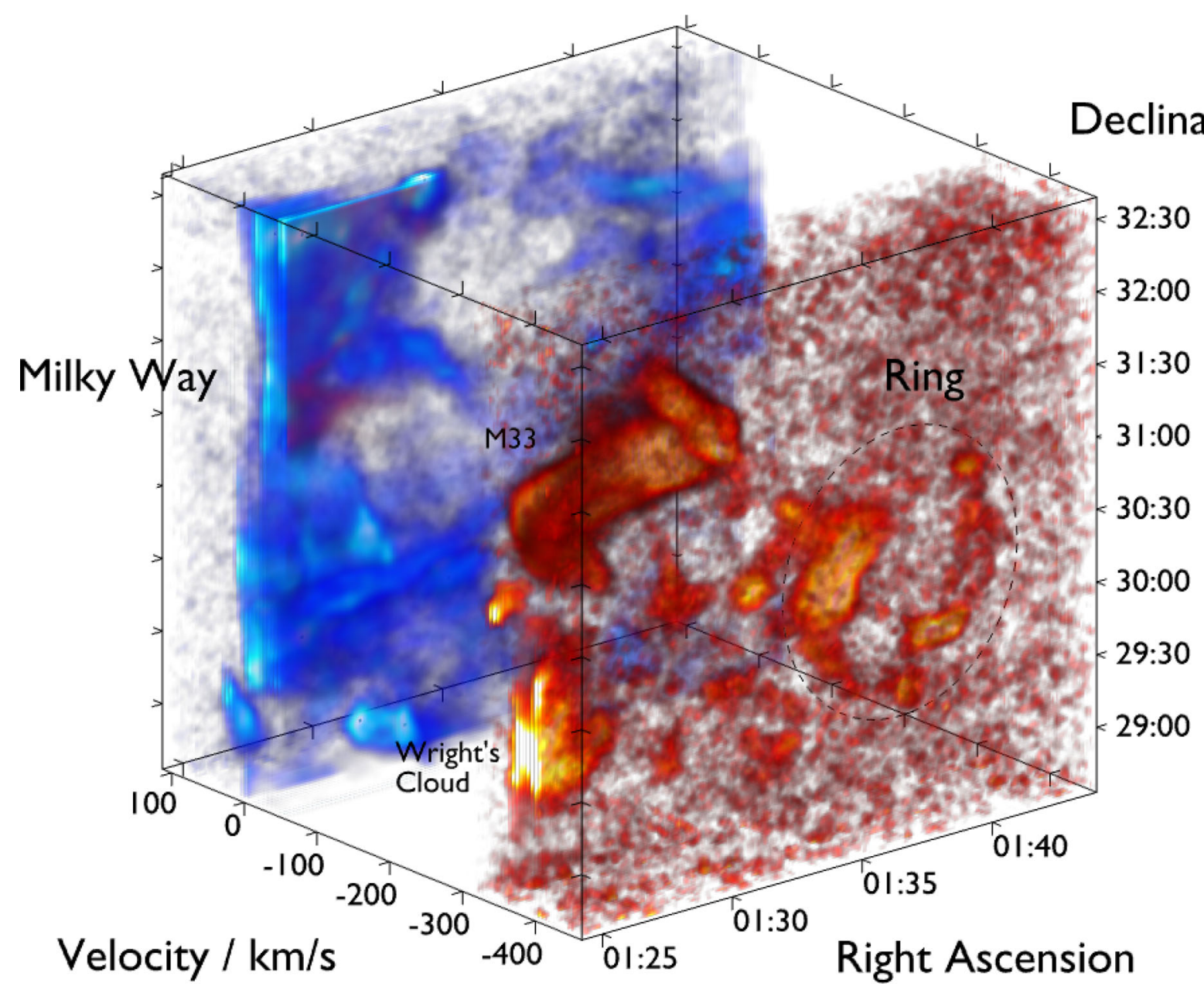

Figure 1. Position-Velocity plot of the AGES Hi data cube. The H I from M33 and the Milky Way are confused over the approximate velocity range of $-75<v<0 \mathrm{~km} \mathrm{~s}^{-1}$ so we have used blue for velocities $v>-75 \mathrm{~km} \mathrm{~s}^{-1}$ (Milky Way) and red for everything else. Some of the distinct $\mathrm{H}$ I structures referenced in the text are labelled. The figure shows just a single, representative still image. A full movie of the rotating cube is available online.

on M33. The full spatial range of the data considered here is from $1^{\mathrm{h}} 23^{\mathrm{m}} 36^{\mathrm{s}}$ to $1^{\mathrm{h}} 44^{\mathrm{m}} 39^{\mathrm{s}}$ in RA, and from $+28^{\circ} 23^{\prime} 50^{\prime \prime}$ to $32^{\circ} 53^{\prime} 54^{\prime \prime}$ in declination (J2000), see Fig. 1.

Observations were performed with the telescope in drift scan mode, which means that the array is kept at a fixed azimuth and elevation whilst the sky drifts overhead. Each drift lasts for $20 \mathrm{~min}$ and covers about 5 deg of RA. The 3.5 arcmin resolution element takes about $13 \mathrm{~s}$ to cross the beam when observing at $1.4 \mathrm{GHz}$, so 25 scans are required for an integration time of $\sim 330 \mathrm{~s}$. Each beam records every second data in two polarizations with 4096 channels, spanning a velocity range of approximately $-2000 \mathrm{~km} \mathrm{~s}^{-1}$ to $+20000 \mathrm{~km} \mathrm{~s}^{-1}$. The two polarizations are combined and then the data are gridded into 1' spatial pixels (the Arecibo beam has an FWHM of 3.5 arcmin at this wavelength) and into velocity channels of width $5.2 \mathrm{~km} \mathrm{~s}^{-1}$.

The data were reduced using the AIPS ++ packages LIVEDATA and GRIDZILLA, which are extensively described in Barnes et al. (2001). In brief, LIVEDATA is used to perform bandpass estimation and removal, Doppler tracking and it calibrates the residual spectrum. We also fit and subtract a sigma-clipped second-order polynomial to each spectrum, which, as shown in Taylor et al. (2014) (hereafter AGES VII), can significantly improve the baseline quality. GRIDZILLA is a gridding package which co-adds all of the spectra to produce the data cube described above. It also performs side lobe correction so that in the final gridded data cube each beam contributes equally to each pixel, with the exception of the edges of the map where sky coverage is incomplete.

We obtain an approximate $1 \sigma \mathrm{H}$ I column density sensitivity of $1.5 \times 10^{17} \mathrm{~cm}^{-2}$ over a velocity of $10 \mathrm{~km} \mathrm{~s}^{-1}$. Our column density sensitivity is around three times better than the ALFALFA data of G08. Sources of any given mass will be detected with lower S/N levels if their velocity width is large or equally, if they have the same intrinsic velocity widths but are discs with higher inclination angles, since their flux is spread out over more channels.

\section{SOURCE EXTRACTION}

To isolate the $\mathrm{H}_{\mathrm{I}}$ associated with M33, we selected a region of the cube spanning a velocity range of $\sim 600 \mathrm{~km} \mathrm{~s}^{-1}$. The systemic velocity of M33 is $\sim-180 \mathrm{~km} \mathrm{~s}^{-1}$ and as the Milky Way is centred at $0 \mathrm{~km} \mathrm{~s}^{-1}$ distinguishing $\mathrm{H}_{\mathrm{I}}$ in M33 from that in the Milky Way is not straightforward (all velocities given are barycentric except where stated otherwise). Each channel was inspected to determine the extent of the Milky Way contamination. H I from the Milky Way appears as extended emission spread across the spatial extent of the cube and is continuously connected, it also appears outside of the region in which one would expect to find M33. The systemic velocities of the two galaxies, along this line of sight, differ by only 
about $200 \mathrm{~km} \mathrm{~s}^{-1}$, which is of the order of their internal velocities, so they 'bleed together' in the cube (Fig. 1). This means that any sources in this crossover area (from around $0 \mathrm{~km} \mathrm{~s}^{-1}$ to $75 \mathrm{~km} \mathrm{~s}^{-1}$ ) must be inspected by eye to determine whether they are likely to be a cloud associated with M33. If we find a compact source as opposed to extended emission across the field then we conclude that the source does not appear to be associated with the Milky Way. This process led to a final data cube analysed here that spanned a velocity range of -500 to $100 \mathrm{~km} \mathrm{~s}^{-1}$, see Fig. 1. This cube is available as an MP4 movie through the online materials. Initially source identification was carried out by visual inspection of the data cube. The cube was split into moment 0 maps each of width $\sim 50 \mathrm{~km} \mathrm{~s}^{-1}$ and these were inspected for individual sources. Visual examination is also preferable to automated source extraction when attempting to distinguish between real sources and radio frequency interference (see Taylor et al. 2013 - AGES VI). However, visual inspection does suffer from our subjectivity and is generally slower than most algorithms. In AGES VII, we describe a new data cube viewer, which has been specifically designed to assist visual extraction of sources from Hi data cubes. ${ }^{1}$ FRELLED was used to visualize and catalogue all of our detections.

We then used the MIRIAD ${ }^{2}$ task MBSPECT to view the spectra of each identified H I cloud and confirm our detections. MBSPECT is particularly useful in this case as it can be used to determine whether a cloud is distinct from the Milky Way (and possibly M33) by confirming that they have separate spectral peaks, i.e. whether our identified H I clouds appear to be distinct both spatially and in velocity. We define a cloud as an object detected after a $3 \sigma$ clip has taken place on the data cube and channel smoothing has been applied (the channels are smoothed to $10 \mathrm{~km} \mathrm{~s}^{-1}$ ). After this the detection is checked with both FRELLED and MBSPECT - for it to be included in our detections it must appear in MBSPECT as a single peak at $3 \sigma$, but may separate into more peaks above this level. MBSPECT was also used to measure the velocity widths (at 50 and 20 per cent of peak flux density) of the identified $\mathrm{H}$ i clouds. We then used the image display and processing package DS9 to fit ellipses to each of the identified sources. From this we obtained the central coordinates of each cloud and the lengths of the semiminor and semimajor axes of the fitted ellipse as measured at a column density of $3 \sigma-$ the noisiest pixel has a $3 \sigma$ noise value of $\sim 0.1 \mathrm{Jy}$. The cloud sizes given were calculated using the measured values of the semimajor and semiminor axes. We used these positions and sizes to measure the total flux density and $\mathrm{H}$ I mass within the detection aperture using the standard formula:

$M=2.36 \times 10^{5} d_{\mathrm{Mpc}}^{2} \sum f_{v} \Delta V$

$d_{\mathrm{Mpc}}$ is the distance to the source in Mpc, which we assume to be the same as M33, i.e. $0.84 \mathrm{Mpc}$ (Freedman et al. 1991), $f_{v}$ is the flux density in Jy and $\Delta V$ is the velocity width in $\mathrm{km} \mathrm{s}^{-1}$. In addition, we can create integrated flux maps by summing pixel values over velocity. Full details of the sources detected and their derived parameters are given in Tables 1 and 2 .

\footnotetext{
${ }^{1}$ The data cube viewer is called FRELLED (FITS Realtime Explorer of Low Latency in Every Dimension). Full details are given in AGES VII appendix $\mathrm{A}$ and the source code is available through our website at www.naic.edu/ ages/.

2 MIRIAD is a radio interferometry data reduction package which can be used for reduction of observations through to image synthesis, analysis and display (Sault, Teuben \& Wright 1995).
}

\section{RESULTS}

As a check of our calibration compared to other surveys, we have measured the $\mathrm{H}$ I mass of M33 in the same way as the other $\mathrm{HI}$ clouds we have identified - we obtain a total $\mathrm{H}$ I mass for M33 of $4.7 \times 10^{9} \mathrm{M}_{\odot}$. To compare our results with a previously derived value, we reduce our aperture to approximately the same size as that given in Putman et al. (2009). When this is done, we obtain a H I mass of $2.0 \times 10^{9} \mathrm{M}_{\odot}$, this compares well with the value given in Putman et al. (2009), which is $1.4 \times 10^{9} \mathrm{M}_{\odot}$ - we agree to within 30 per cent, though defining exactly the same apertures both spatially and in velocity is not possible.

Using the source extraction methods described in Section 3, we have identified 32 clouds that have a probable association with M33. In the appendix we show moment zero (integrated flux maps), moment 1 (velocity field) and spectra for each detected source. The sources are split into two groups: those that appear discrete and those that clearly show an extended connection to M33. One clear initial result is that some of the features identified by G08 as discrete clouds are actually connected to the $\mathrm{H}$ I disc of M33 by areas of low column density $\mathrm{H}_{\mathrm{I}}$ in our data, which has a better column density sensitivity. This is demonstrated in Fig. 2 which shows the positions of clouds, which fall within the disc of M33.

We employ a similar distinguishing criterion as Braun \& Thilker (2004) to separate what we describe as discrete objects from those that are clearly linked to M33 by low column density H I, i.e. they describe them as discrete if there is no connection observed down to a column density of $1.5 \times 10^{17} \mathrm{~cm}^{-2}$. Table 1 is a full list of the $22 \mathrm{H} \mathrm{I}$ clouds that we identify as being discrete down to our column density sensitivity limit of $\sim 1.5 \times 10^{17} \mathrm{~cm}^{-2}$. Table 2 lists 10 additional clouds from G08, which we detect but are either clearly part of the disc of M33 or joined to M33 by low column density H. These latter objects have been listed in a separate table as we argue that they cannot be classified as independent clouds due to their obvious connection to M33. These objects may be of an entirely different nature to those in Table 1, for example the result of tidal interactions rather than potential primordial objects. Of course, subsequent even better sensitivity observations may reveal even more connectivity between objects we consider to be discrete.

We detect all clouds listed in G08, with the exception of their clouds AA3, AA22 and AA23, which do not appear in our data at the coordinates and velocities specified. G08 state that their clouds AA4, AA6, AA12, AA13 and AA14 are connected to M33, we extend this list to include AA7, AA8, AA11, AA15 and AA16. In addition to this, the clouds G08 designate as AA21a and AA21b appear in our data to be a single ring-like structure (AGESM33-31, see below). A moment zero map of our data is shown in Fig. 3. The outline of M33's H I disc is shown, with all discrete clouds overlaid. The figure was made by integrating the flux over the velocity range of each cloud, then filling in the lowest contour. The colour indicates the central velocity of each cloud. The large structure to the bottom right of Fig. 3 has previously been identified and is known as Wright's cloud (Wright 1979).

We can also compare our results with those of BT04. As stated above their survey covers the much larger M33/M31 region, but is somewhat compromised compared to our survey by the large spatial resolution of 49 arcmin. Using the BT0 4 sensitivity limits, we calculate that their minimum detectable $\mathrm{H}_{\mathrm{I}}$ mass at the distance of M33 is about $10^{5} \mathrm{M}_{\odot}$, which is smaller than the mass of most of the clouds we detect (see Tables 1 and 2). BT04 only detect two discrete clouds over the region where our data overlaps (as listed in Thilker, Braun \& Walterbos 2002), so the limitation of their data 
Table 1. The above table gives our derived properties for all 'isolated' clouds. The columns are: (1) name, (2) and (3) co-ordinates in right ascension and declination (J2000), (4) the velocity width at the FWHM (50 per cent), (5) the central velocity of the cloud given by MBSPECT, (6) the velocity relative to M33 (the velocity of M33 is taken to be $-180 \mathrm{~km} \mathrm{~s}^{-1}$, (7) the H i mass at the distance of M33, (8) the total measured flux density, (9) the measured size of the cloud along the semimajor and semiminor axes in kpc. These were measured to the nearest pixel which corresponds to $\sim 0.2 \mathrm{kpc},(10)$ the peak column density of the cloud as measured by our 3.5 arcmin beam and (11) whether the cloud has been previously detected by another survey. N.B. values given for AGESM33-32 (Wright's cloud) only measure the amount of the cloud visible in our cube and not the entirety. Where necessary a distance to the clouds of $840 \mathrm{kpc}$ is assumed (Freedman et al. 1991). The errors on velocities and fluxes were calculated with parameters from MBSPECT using equations given in Auld et al. (2006). Where the error on the velocity was calculated to be $<3 \mathrm{~km} \mathrm{~s}^{-1}$ the error is given as $3 \mathrm{~km} \mathrm{~s}^{-1}$. This is because the central velocity is accurate to with half a channel.

\begin{tabular}{|c|c|c|c|c|c|c|c|c|c|c|}
\hline AGES ID & $\begin{array}{c}\text { RA } \\
(\mathrm{J} 2000) \\
(2)\end{array}$ & $\begin{array}{c}\text { Dec } \\
(\mathrm{J} 2000) \\
(3)\end{array}$ & $\begin{array}{c}\text { Velocity } \\
\text { width 50 } \\
\left(\mathrm{km} \mathrm{s}^{-1}\right) \\
(4)\end{array}$ & $\begin{array}{c}\text { Systemic } \\
\text { velocity } \\
\left(\mathrm{km} \mathrm{s}^{-1}\right) \\
(5)\end{array}$ & $\begin{array}{l}\text { Velocity } \\
\text { relative to M33 } \\
\left(\mathrm{km} \mathrm{s}^{-1}\right) \\
(6)\end{array}$ & $\begin{array}{c}\text { Н I Mass } \\
\left(\mathrm{M}_{\odot}\right) \\
(7)\end{array}$ & 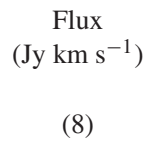 & $\begin{array}{c}\text { Size } \\
(\mathrm{a}, \mathrm{b} \mathrm{kpc}) \\
(9)\end{array}$ & $\begin{array}{c}\text { Column } \\
\text { density } \\
\left(\mathrm{cm}^{-2}\right) \\
(10)\end{array}$ & $\begin{array}{c}\text { Previous } \\
\text { detection? } \\
\text { (11) }\end{array}$ \\
\hline AGESM33-1 & $01: 34: 51.3$ & $+30: 59: 59$ & 51.0 & $-86 \pm 3$ & $94 \pm 3$ & $1.18 \times 10^{6}$ & $7.1 \pm 0.4$ & $3.0 \times 1.7$ & $3.9 \times 10^{18}$ & G08-AA1 \\
\hline AGESM33-2 & $01: 40: 13.2$ & $+30: 50: 57$ & 26.0 & $-111 \pm 15$ & $69 \pm 15$ & $1.62 \times 10^{6}$ & $9.7 \pm 2.5$ & $4.9 \times 2.4$ & $2.4 \times 10^{18}$ & G08-AA2 \\
\hline AGESM33-3 & 01:44:03.7 & $+31: 14: 33$ & 30.5 & $-121 \pm 3$ & $59 \pm 3$ & $1.48 \times 10^{6}$ & $8.9 \pm 0.2$ & $3.7 \times 2.4$ & $2.9 \times 10^{18}$ & None \\
\hline AGESM33-5 & $01: 39: 31.4$ & $+30: 41: 30$ & 32.8 & $-126 \pm 3$ & $54 \pm 3$ & $2.13 \times 10^{6}$ & $12.8 \pm 0.2$ & $2.4 \times 2.4$ & $6.2 \times 10^{18}$ & G08-AA5 \\
\hline AGESM33-10 & 01:41:00.8 & $+30: 05: 17$ & 21.0 & $-164 \pm 3$ & $16 \pm 3$ & $2.33 \times 10^{5}$ & $1.4 \pm 0.1$ & $2.4 \times 2.4$ & $6.9 \times 10^{17}$ & None \\
\hline AGESM33-12 & $01: 33: 27.8$ & $+29: 14: 49$ & 29.2 & $-186 \pm 4$ & $-6 \pm 4$ & $6.66 \times 10^{5}$ & $4.0 \pm 0.3$ & $3.7 \times 2.4$ & $1.3 \times 10^{18}$ & G08-AA9 \\
\hline AGESM33-14 & $01: 31: 18.3$ & $+30: 00: 54$ & 22.7 & $-188 \pm 3$ & $-8 \pm 3$ & $1.91 \times 10^{6}$ & $11.5 \pm 0.2$ & $1.7 \times 1.7$ & $1.1 \times 10^{19}$ & G08-AA10 \\
\hline AGESM33-19 & $01: 29: 52.0$ & $+31: 04: 23$ & 34.1 & $-288 \pm 3$ & $-108 \pm 3$ & $9.99 \times 10^{4}$ & $0.6 \pm 0.1$ & $3.7 \times 1.2$ & $3.5 \times 10^{17}$ & G08-AA17 \\
\hline AGESM33-20 & $01: 34: 27.6$ & $+29: 30: 00$ & 38.4 & $-315 \pm 6$ & $-135 \pm 6$ & $2.00 \times 10^{6}$ & $12.0 \pm 0.5$ & $6.1 \times 3.7$ & $1.6 \times 10^{18}$ & None \\
\hline AGESM33-21 & $01: 39: 41.7$ & $\begin{array}{l}+29: 58: 32 \\
\end{array}$ & 32.6 & $-307 \pm 5$ & $-127 \pm 5$ & $1.67 \times 10^{5}$ & $1.0 \pm 0.1$ & $1.7 \times 1.7$ & $1.0 \times 10^{18}$ & None \\
\hline AGESM33-26 & $01: 24: 32.8$ & $+30: 36: 43$ & 56.4 & $-359 \pm 5$ & $-179 \pm 5$ & $5.99 \times 10^{5}$ & $3.6 \pm 0.2$ & $3.2 \times 2.4$ & $1.3 \times 10^{18}$ & None \\
\hline AGESM33-27 & $01: 27: 17.8$ & $+30: 05: 21$ & 16.9 & $-377 \pm 3$ & $-197 \pm 3$ & $6.66 \times 10^{5}$ & $4.0 \pm 0.2$ & $7.3 \times 2.4$ & $6.5 \times 10^{17}$ & None \\
\hline AGESM33-28 & 01:38:04.7 & $+29: 56: 46$ & 44.8 & $-335 \pm 5$ & $-155 \pm 5$ & $2.26 \times 10^{6}$ & $13.6 \pm 0.5$ & $9.7 \times 3.7$ & $1.1 \times 10^{18}$ & None \\
\hline AGESM33-29 & $01: 33: 18.6$ & $+29: 29: 59$ & 71.1 & $-335 \pm 7$ & $-155 \pm 7$ & $5.16 \times 10^{5}$ & $3.1 \pm 0.2$ & $3.7 \times 2.4$ & $9.9 \times 10^{17}$ & G08-AA19 \\
\hline AGESM33-30 & $01: 40: 20.3$ & $+29: 11: 24$ & 24.5 & $-341 \pm 3$ & $-161 \pm 3$ & $5.00 \times 10^{5}$ & $3.0 \pm 0.2$ & $3.7 \times 2.4$ & $9.8 \times 10^{17}$ & None \\
\hline AGESM33-31 & $01: 37: 00: 2$ & $+30: 01: 52$ & 17.7 & $-374 \pm 3$ & $-194 \pm 3$ & $1.22 \times 10^{7}$ & $73.5 \pm 0.5$ & $18.2 \times 14.6$ & $7.9 \times 10^{17}$ & G08-AA21a, 21b \\
\hline AGESM33-32 & $01: 24: 06.1$ & $+29: 38: 35$ & 25.4 & $-383 \pm 3$ & $-203 \pm 3$ & $4.54 \times 10^{7}$ & $272.6 \pm 0.7$ & $10.9 \times 10.9$ & $6.5 \times 10^{18}$ & Wright's cloud \\
\hline
\end{tabular}

Table 2. The above table gives our derived properties of all clouds listed by Grossi et al 2008 which are either part of the disc of M33 or joined to M33 by low column density H I. All columns are the same as in Table 1, with the exception of column 11 which is the cloud ID given in G08.

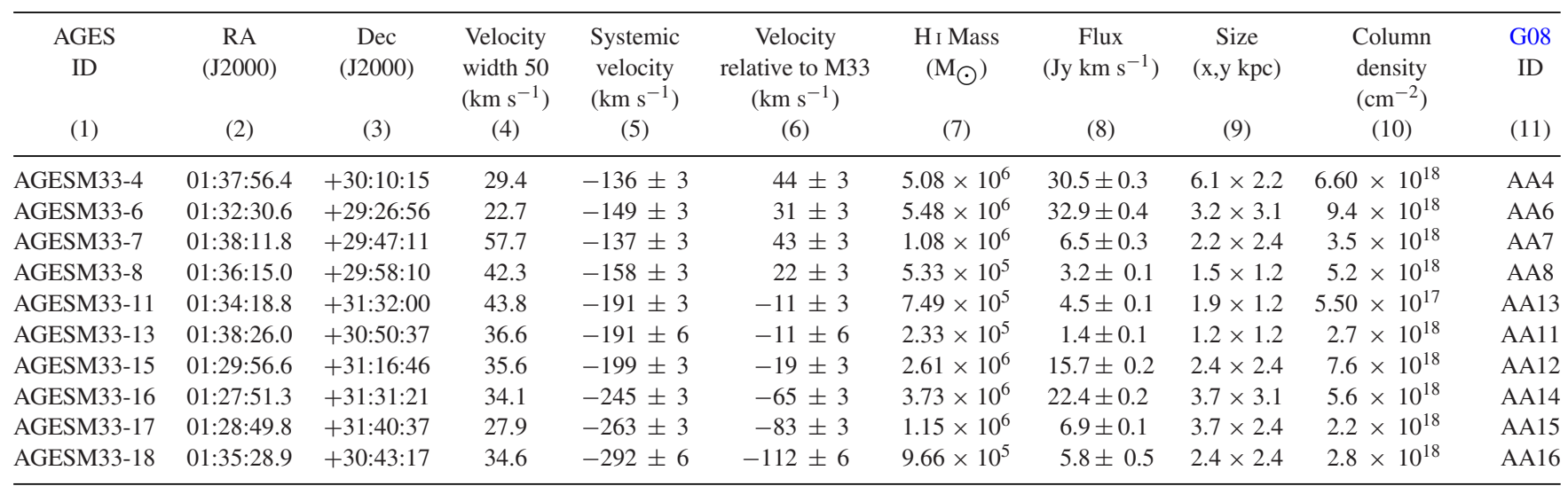

compared to ours must be their spatial resolution. We detect both of the clouds described in BT04 using the AGES data. Wright's cloud (AGESM33-32), which is a previously known H I cloud, is either associated with M33 or possibly extended debris from the Magellanic stream. Wright's cloud has a total mass of $\sim 10^{8} \mathrm{M}_{\odot}$ (Wright 1979), but our cube only contains part of it (Fig. 3), it is fully described in Wright 1979. The second cloud, AGESM33-31, will be discussed in detail in Section 5.

The clouds we detect range in $\mathrm{HI}$ mass from $1.0 \times 10^{5} \mathrm{M}_{\odot}$ to $4.5 \times 10^{7} \mathrm{M}_{\odot}$ and have velocity widths of between $\sim 17$ and $\sim 72 \mathrm{~km} \mathrm{~s}^{-1}$ at 50 percent of their peak flux density. The smallest of the clouds is just over $1 \mathrm{kpc}$ in size and, as the Arecibo beam size is about $1 \mathrm{kpc}$ on the sky at the distance of M33 $(840 \mathrm{kpc})$, we would not expect to measure sizes any smaller than this.

\section{DISCUSSION}

We have identified $32 \mathrm{H}$ I structures around the nearby galaxy M33. 22 of these appear to be discrete clouds (Table 1), while a further 


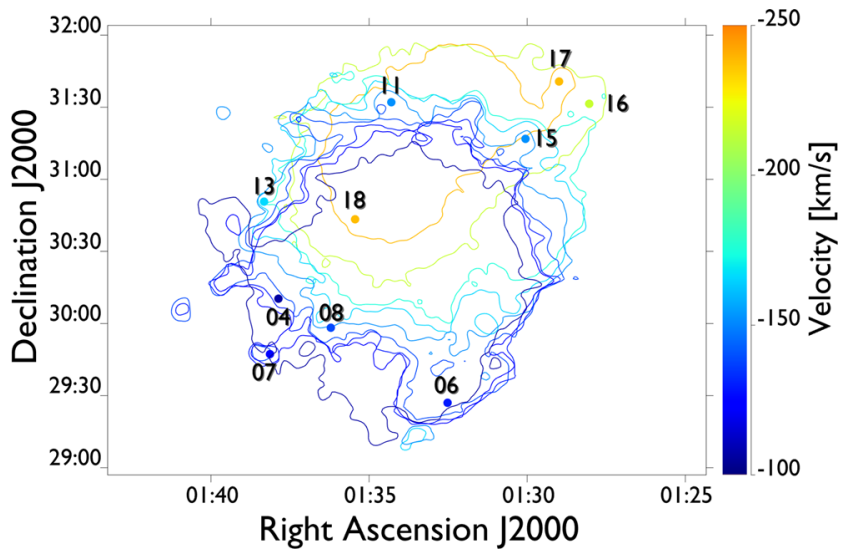

Figure 2. A Renzogram of M33 across a velocity range of $150 \mathrm{~km} \mathrm{~s}^{-1}$. Labelled dots indicate the positions of clouds which appear to form part of the disc of M33 (Table 2). Colours are indicative of velocity.

10 appear to have low column density structures that connect them to M33 (Fig. 2). 11 of the clouds appear to be previously undetected which is due to our area coverage, sensitivity and resolution compared to other surveys.

The $\mathrm{H}_{\mathrm{I}}$ masses of known dwarf galaxies in the Local Group range from $1.2 \times 10^{5}$ to $1.8 \times 10^{8} \mathrm{M}_{\odot}$ (data from tables 1 and 4 in McConnachie 2012), which means that the majority of our detected $\mathrm{H}$ I clouds have $\mathrm{H}$ I masses that are typical of known dwarf galaxies. If our detected objects are progenitor dwarf galaxies that currently lack a stellar component then a better comparison is with the total baryonic mass of other Local Group dwarf galaxies. The baryonic masses of Local Group dwarfs range from $3.4 \times 10^{2} \mathrm{M}_{\odot}$ to $4.6 \times 10^{9} \mathrm{M}_{\odot}$ (also calculated using data in McConnachie 2012 by multiplying the H I mass by 1.4 to account for the primordial fraction of helium and then adding the stellar mass to get the total baryonic mass, where no $\mathrm{H}$ I mass is given only stellar mass is used). In Fig. 4, we show a comparison of these baryonic masses with the H I masses of our detections - clearly our detections have masses consistent with those of dwarf galaxies, though at the lower end of the mass scale.

As stated in the Introduction, it has been suggested that there may be a link between Galactic HVCs, dwarf galaxies and the missing subhalo population (Blitz et al. 1999, 2004). It has also been suggested that a particular class of HVC, known as compact HVC or CHVC, may actually be part of the missing subhalo population (Braun \& Burton 1999; Blitz et al. 1999). We can compare our discrete $\mathrm{H}$ I clouds to CHVCs detected around the Milky Way. Burton, Braun \& Chengalur (2001), who also used the Arecibo telescope, specifically targeted 10 Milky Way CHVCs. They measured their central Hi column densities to be in the range $2 \times$ $10^{19}-2 \times 10^{20} \mathrm{~cm}^{-2}$ and typical line-of-sight velocity dispersions of $\sim 11 \mathrm{~km} \mathrm{~s}^{-1}$. Sternberg et al. (2002) describe CHVCs as having average column densities of $\sim 5 \times 10^{18} \mathrm{~cm}^{-2}$ and typical line-ofsight velocity dispersions of $\sim 14 \mathrm{~km} \mathrm{~s}^{-1}$. Comparing these values with those given in Table 1 clearly shows that our $\mathrm{H}_{\mathrm{I}}$ detections are different to Milky Way CHVCs. Our detections generally have lower peak H i column densities (as measured by our 3.5 arcmin

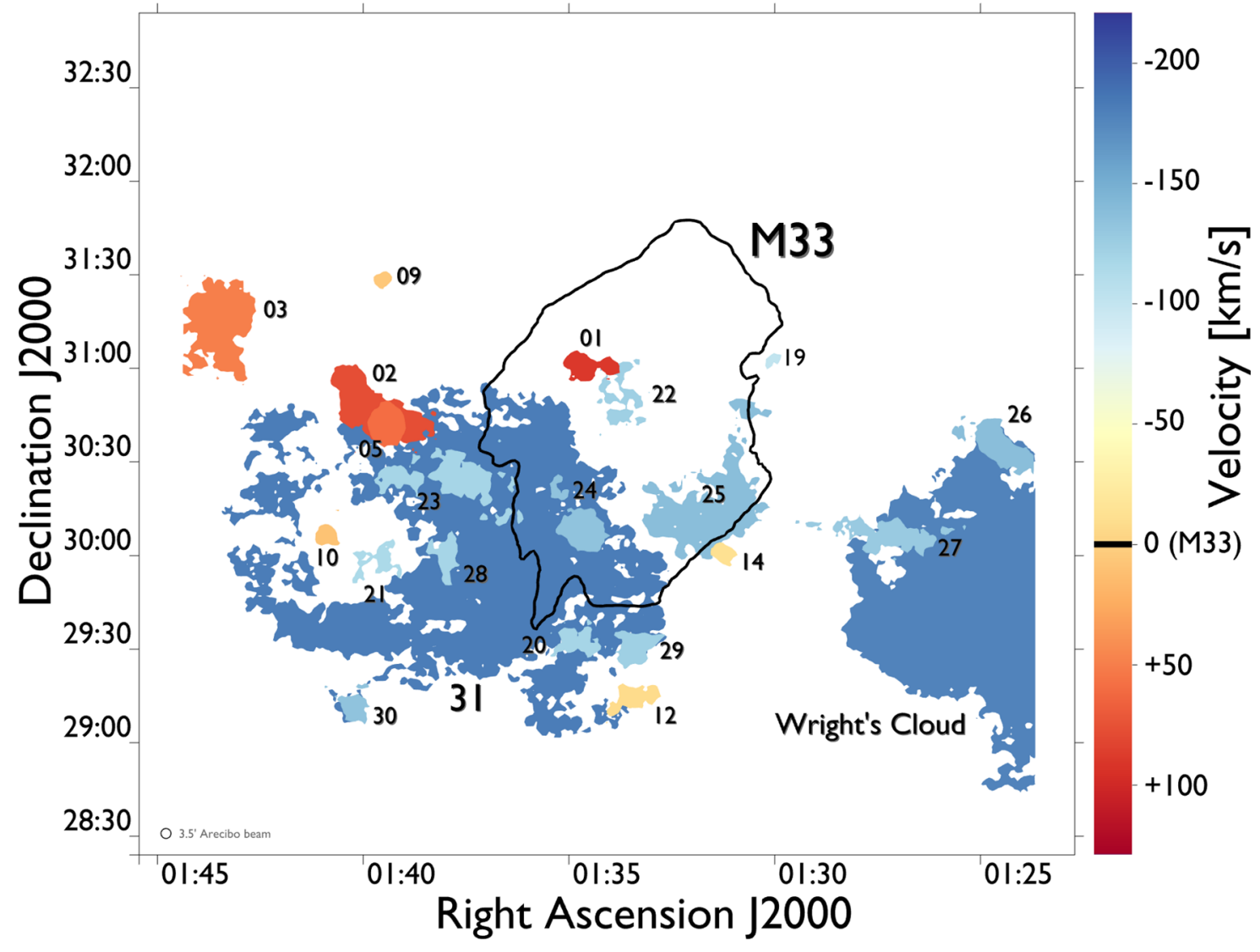

Figure 3. The outline of M33's H I disc is shown, with all of the discrete detected clouds overlaid. The figure was made by integrating the flux over the velocity range of each cloud, then filling in the lowest contour. The central velocity of each cloud was used so the colours are indicative of this velocity. The black line shows the approximate extent of M33. Velocities shown are relative to M33. 


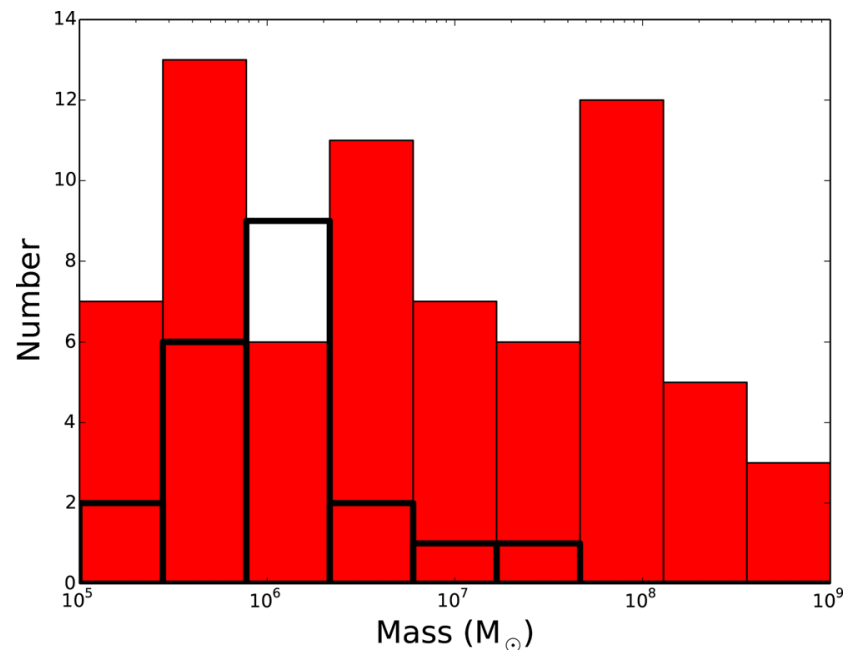

Figure 4. A histogram showing the baryonic masses of our detected $\mathrm{HI}$ clouds (black outline) compared to Local Group dwarf galaxies (red). The baryonic masses of the Local Group dwarf galaxies were obtained from table 4 in McConnachie 2012 - from this table, we have multiplied the $\mathrm{H}_{\mathrm{I}}$ mass by 1.4 to account for the primordial fraction of helium and then added the stellar mass to get the total baryonic mass (this ignores molecular and ionized gas).

beam) and larger velocity widths, values more typical of HVCs rather than CHVCs.

Both clouds AGESM33-31 and AGESM33-32 are especially interesting as they are both extremely extended spatially compared to the other clouds detected and have comparably small velocity widths for their extent. This is particularly so for AGESM33-31, which has the second smallest velocity width of all of our detections. AGESM33-31 was detected by G08 as AA21a and AA21b, however in our data it appears as a single much larger ring-like structure (see Figs 1, 3 and 5). It was also previously detected by Thilker et al. (2002) and designated 'M33 CHVC' but was unresolved in their survey. So, this is the first time that this object has been observed as a ring-like structure. It is extremely spatially extended, similar to Wright's cloud (seen in our data as AGESM33-32, Fig. 3), and also occupies almost the same velocity range. It has been suggested that Wright's cloud is associated with the Magellanic stream due to an extended low $N_{\mathrm{HI}}$ component of the cloud that extends in the direction of the stream (e.g. Nidever et al. 2010), however it is of the order of $50 \mathrm{kpc}$ away from the main structure of the stream and $\sim 30 \mathrm{kpc}$ from the S0 filament of the stream (as defined by Nidever et al. 2010). It is possible that AGESM33-31 is also in some way related to the Magellanic stream, rather than being an HVC of M33, though it is even further away from the main section of the Magellanic stream than Wright's cloud. ${ }^{3}$ So, a Magellanic stream origin is a possibility, but another interpretation is that AGESM33-31 is a Galactic HVC projected along this line of sight. At the distance of M33 AGESM33-31 has a rather large size of $\sim 18 \mathrm{kpc}$ (bigger than M33) but a rather low Hi mass about 1 per cent of that in M33. This in itself may be just the characteristics of the 'failed' galaxies we have been looking for, but of course if AGESM33-31 was much closer to the Galaxy the $\mathrm{H}_{\text {I }}$ mass and size would reduce to something much more like an HVC - so this is another possibility that cannot be ruled out.

${ }^{3}$ As we only observe a part of Wright's cloud, we will not discuss it further here.
Being a little more speculative and accepting a location close to M33, AGESM33-31 appears to be a rather large $\mathrm{H}$ I cloud with a big hole in it. A feature like this is plausibly tidal in origin, however, if this was $\mathrm{H}_{\mathrm{I}}$ in a known galaxy its appearance could be interpreted as a void in the $\mathrm{H}_{\mathrm{I}}$ vacated by a stellar wind or supernova explosion (e.g. Oey et al. 2002). Thilker et al. (2002) identify similar H I 'holes' and 'shells' in the disc of M33, but with a size of $\sim 10 \mathrm{kpc}$ the hole in AGESM33-31 is about an order of magnitude larger than that normally thought to have resulted from stellar winds. Of course a hole created by a stellar wind implies stars and so a link between these $\mathrm{H}_{\mathrm{I}}$ clouds and what more traditionally has been described as a galaxy. ${ }^{4}$ Further detailed investigation of the distribution of stars across this area will have to await the release of data from surveys such as PAndAS CUBS (e.g. Martin et al. 2009). However, continuing this 'galaxy' theme the very low measured velocity width $\left(18 \mathrm{~km} \mathrm{~s}^{-1}\right)$ is consistent with the velocity dispersion of atomic gas seen perpendicular to the plane of a typical disc galaxy (of the order of $10 \mathrm{~km} \mathrm{~s}^{-1}$, Putman et al. 2009 give a velocity dispersion for the atomic hydrogen in M33 of $19 \mathrm{~km} \mathrm{~s}^{-1}$ ) - it may be possible that we are seeing a face-on disc of $\mathrm{H}_{\mathrm{I}}$. In Fig. 5(b), we show the velocity field for AGESM33-31, there is clearly a velocity gradient across the ring of about $30 \mathrm{~km} \mathrm{~s}^{-1}$, but whether this is due to rotation or some form of streaming motion is unclear. Subsequently we found that similar ideas have been reached by Thilker et al. (2002) they describe AGESM33-31 (M33CHVC) as a 'dark companion' to M33.

One striking feature of Table 1 is that clouds AGESM33-23 to 30 all lie at very similar velocities. This is all the more remarkable because spatially they extend some $4^{\circ}$ or about $60 \mathrm{kpc}$ across the sky (Fig. 6). The clouds also have velocities not too dissimilar to that of AGESM33-31 and 32 discussed above (differ by about $40 \mathrm{~km} \mathrm{~s}^{-1}$ ). This raises the question as to whether they are all part of some larger structure for which we are only detecting the higher column density parts and that it is possibly debris from the Magellanic stream as discussed above. We currently list them as separate clouds, but their similar velocities are intriguing.

The possibility of this larger structure could explain a very unisotropic distribution of clouds in the vicinity of M33 - they are concentrated in a band roughly to the south of M33. In addition, the distribution of line-of-sight velocities (see below) is also highly anisotropic - 14 out of 22 clouds have velocities between -100 and $-200 \mathrm{~km} \mathrm{~s}^{-1}$ relative to M33. Both the spatial and velocity distributions are different from what is expected for a population of cold dark matter (CDM) satellites residing in the halo of M33, unless it has been significantly disturbed in a tidal interaction with M31.

Given that we detect 22 discrete $\mathrm{H}$ I clouds around M33, we now consider whether the number of clouds found is consistent with galaxy formation models. We assume that the detected clouds act as tracers of the dark matter haloes that the models predict. Klypin et al. (1999) have used a numerical simulation, which implements the $\Lambda \mathrm{CDM}$ cosmology, to model the dark matter subhalo population around individual galaxies. Sternberg, McKee \& Wolfire (2002) have parametrized the results of Klypin et al. (1999) into the useful

\footnotetext{
${ }^{4}$ Huxor et al. (2009) identify a globular cluster, HM33-B, which is within the coordinate range of AGESM33-31. As no mass or distance is given for this cluster, we cannot draw any further conclusions here. Grossi et al. (2011) have carried out follow-up observations of regions around M33 searching for stellar populations. They report no evidence for a young stellar population in their clouds and hence conclude that there has been no in situ star formation.
} 


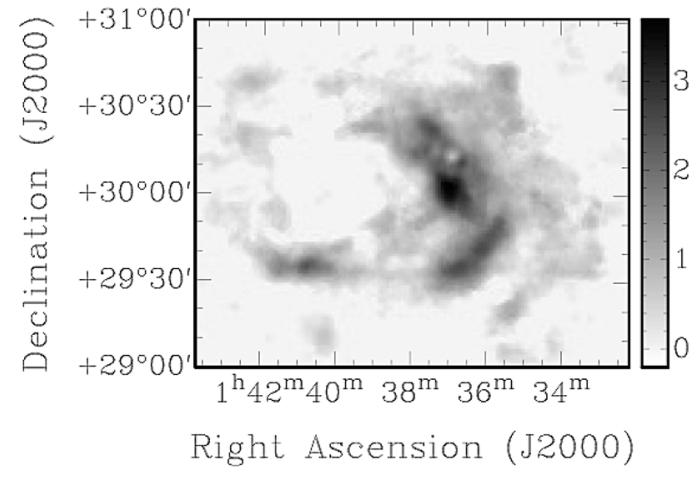

(a)

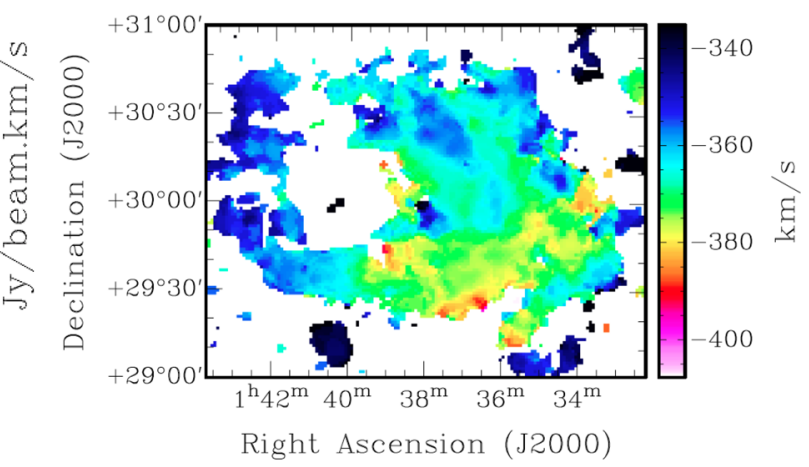

(b)

Figure 5. (a) A H I integrated flux map and (b) a velocity map of AGESM33-31. The cloud is listed as two distinct objects by G08, but it is clearly a ring-like structure. The velocity map shows the cloud has a velocity gradient across it of about $30 \mathrm{~km} \mathrm{~s}^{-1}$. To convert the integrated flux map to a column density map the conversion factor is $2.5 \times 10^{19} \mathrm{~cm}^{-2}\left(\mathrm{Jy} \mathrm{beam}^{-1} \mathrm{~km} \mathrm{~s}^{-1}\right)^{-1}$.

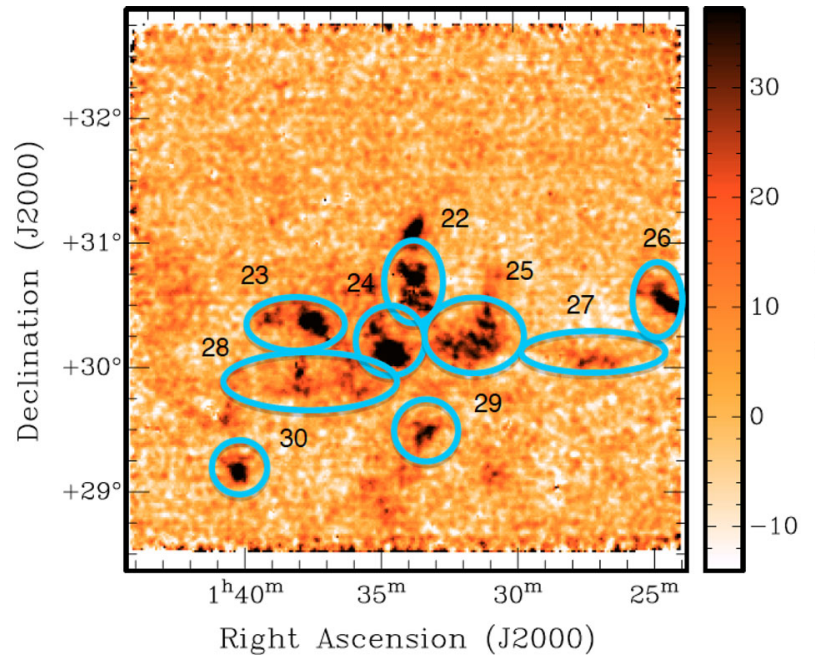

Figure 6. An integrated flux map of a section of the cube over the full RA and Dec. range, but from -320 to $-346 \mathrm{~km} \mathrm{~s}^{-1}$ in velocity, showing a group of clouds with very similar central velocities. AGESM33 cloud numbers are shown. The uncircled patch above cloud 22 is the northern arc of M33.

equation given below that can be used to predict the number of dark matter haloes expected.

$$
\begin{aligned}
& N\left(>v_{s},<d\right) \\
& \quad=1.06 \times 10^{3}\left(\frac{M_{\text {vir }, \mathrm{p}}}{10^{12} \mathrm{M}_{\odot}}\right)\left(\frac{v_{\mathrm{s}}}{10 \mathrm{~km} \mathrm{~s}^{-1}}\right)^{-2.75}\left(\frac{d}{1 \mathrm{Mpc}}\right) .
\end{aligned}
$$

Where $N\left(>v_{\mathrm{s}},<d\right)$ is the total number of subhaloes with scale (circular) velocities greater than $v_{\mathrm{s}}$, which we take to be $\frac{\Delta w_{50}}{2}$, contained within a distance $d$ from the centre of a parent halo, $M_{\mathrm{vir}, \mathrm{p}}^{2}$ is the virial mass of the parent galaxy. For the virial mass of M33 we use $5 \times 10^{11} \mathrm{M}_{\odot}$ (Corbelli 2003). We observe out to $\sim 2.25$ from M33, which corresponds to a distance of about $33 \mathrm{kpc}$ at a distance of $840 \mathrm{kpc}$. Assuming that we have detected all Hi clouds with velocities greater than our minimum detected velocity $\left(\Delta v_{50}\right)$ we have $v_{\mathrm{s}} \approx 9 \mathrm{~km} \mathrm{~s}^{-1}$. Putting these numbers into the above equation the model predicts $\sim 25$ subhaloes. This compares remarkably well with the $22 \mathrm{H}$ i clouds detected. We can actually do a little better than this and compare the distribution of subhalo velocities widths with that predicted by the model (Fig. 7). A Kolmogorov-Smirnov test on the data shown in Fig. 7 gives a KS statistic of 0.166 and a

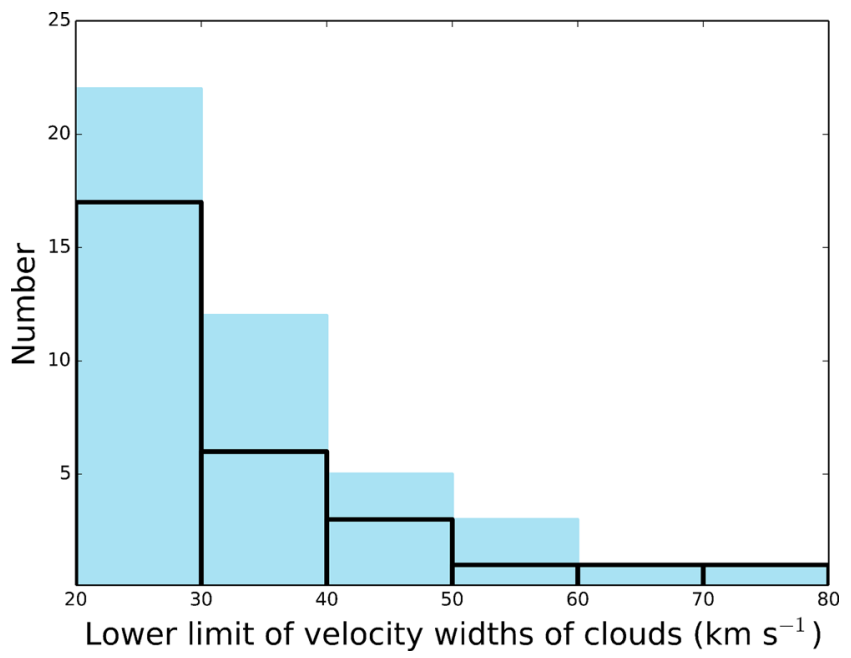

Figure 7. The above shows the number of clouds with velocity widths greater than a given value. The black edged bars are the number of clouds predicted from the numerical simulation described in the text. The blue bars are for the discrete AGESM33 $\mathrm{H}$ i clouds we observe.

$P$-value of 0.999 indicating that we cannot reject the hypothesis that the two samples are from the same distribution. In summary, the numbers of $\mathrm{H}$ I clouds and the distribution of their velocity widths is consistent with the properties of subhaloes produced in numerical cosmological simulations.

However, the main issue with the above calculation is whether the 'observed' velocities can be used as a measure of the mass of the halo that the $\mathrm{H}$ I cloud might reside within. One distinguishing feature of dwarf galaxies is their very high mass-to-light ratios and hence the inference of large quantities of dark matter (McConnachie 2012). This inference of course also assumes that dwarf galaxies are gravitationally bound and that they are supported against collapse by either ordered rotation or velocity dispersion. Both our $\mathrm{H}$ I clouds and other dwarf galaxies within the Local Group may have the velocities of both their gas and stars severely affected by other streaming (possibly tidal) motions, thus rendering mass-to-light calculations invalid.

To investigate this further, we have created moment 1 (velocity) maps - these maps display the velocity range across each object. The maps are shown in Appendix A along with integrated flux maps and spectra. Our conclusion after inspecting the velocity maps is 
that with the possible exception of cloud AGESM33-27 and the comments already made about AGESM33-31, there is little or no evidence of ordered rotation within these clouds.

Although we find little evidence for rotation, it is still possible that the clouds are supported by velocity dispersion, in which case we make a tentative estimate of their dynamical masses $\left(M_{\text {Dyn }}\right)$. We have done this by simply assuming spherical virialized clouds (Blitz et al. 1999), that the FWHM of the velocity profile $\left(\Delta v_{50}\right)$ is a sufficiently accurate estimate of the $3 \mathrm{D}$ velocity dispersion of the gas and that the geometric mean of the measured semiminor and semimajor axis $\left(r_{\mathrm{Geo}}\right)$ is the size of the $\mathrm{H}_{\mathrm{I}}$ cloud. $M_{\mathrm{Dyn}}$ can then simply be approximated using

$M_{\text {Dyn }}=\Delta v_{50}^{2} r_{\mathrm{Geo}} / G$.

Derived dynamical masses range between $10^{9}$ and $10^{11} \mathrm{M}_{\odot}$ and hence mass-to-light ratios would typically need to be of the order of $10^{4}$ for these objects to be bound. We find no correlation between $\mathrm{H}$ I and dynamical masses within our detections, which would be expected if they had a constant ratio of dark to baryonic matter.

These very high mass-to-light ratios assume that we have detected all of the hydrogen gas present in the cloud. Sternberg et al. (2002) have modelled $\mathrm{H}$ I clouds immersed in an ionizing background as might be expected if they reside close to a star-forming galaxy like M33. They conclude that the $\mathrm{H}_{\mathrm{I}}$ remains neutral for column densities greater than about $10^{19} \mathrm{~cm}^{-2}$ and that for typical HVCs only 10 per cent may remain in the neutral form, i.e. there may be as much as 10 times more hydrogen present than is inferred from $21 \mathrm{~cm}$ observations. We would still require mass-to-light ratios of the order of $10^{3}$ for bound objects, which although high, is not totally inconsistent with observations of Local Group dwarf galaxies (McConnachie 2012).

Fig. 3 shows the detected H clouds with colours to indicate their velocities relative to M33. A test of whether the clouds we have described as 'discrete' are a different population to those that have an apparent low column density connection to M33, is their comparative distribution of velocities. Fig. 3 clearly shows that the discrete clouds are biased towards higher velocities in relation to M33 and could be described as its HVC population. On the other hand, Fig. 2 clearly shows that those objects with a low column density connection to M33 are also connected in velocity space. Fig. 2 is a renzogram, which is a plot of contours of M33 at different velocities - the objects listed in Table 2 fall within these contours at their specific velocity - this does not happen for the discrete objects listed in Table 1. We feel that both Figs 2 and 8 indicate that we have been able to separate discrete $\mathrm{H}_{\mathrm{I}}$ structures from those that have an origin within the disc of M33.

\section{SUMMARY}

In this paper, we have utilized deep $21 \mathrm{~cm}$ Arecibo data from the AGES survey to investigate the nature of the halo around M33. Our particular interest lies in the lack of previously identified dwarf galaxy (subhaloes) companions of M33, something that at first sight is counter to expectations from galaxy formation models. We identify 22 discrete hydrogen clouds that have a distribution of internal velocity dispersions consistent with expectations from standard galaxy formation models. However, the issue remains whether the observed velocity dispersions can be used as a measure of the $\mathrm{H}$ I clouds total mass, i.e. are the velocities indicative of virialized structures or have they been influenced by tidal interactions with other structures in the Local Group. If the latter is true then they probably cannot be associated with the missing subhalo population. We

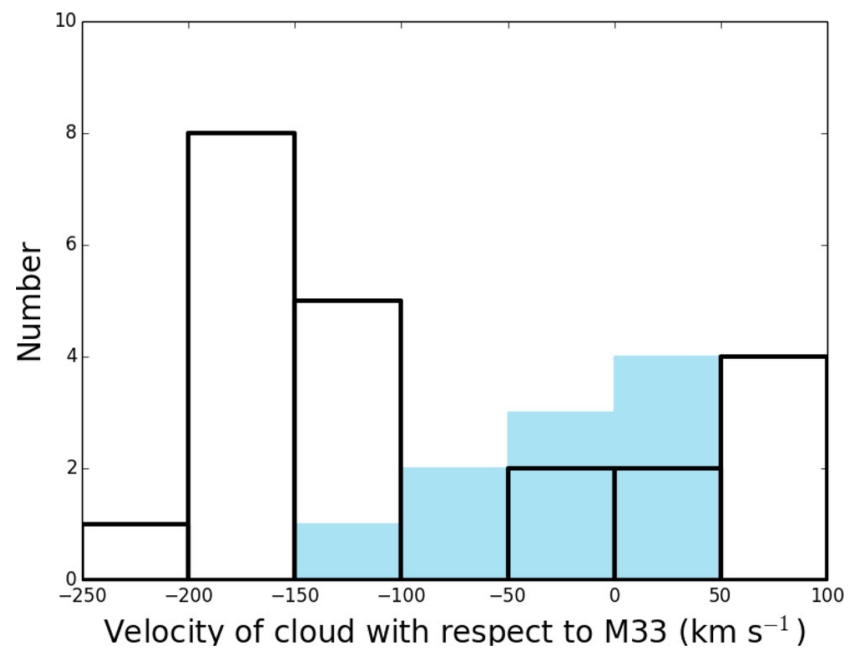

Figure 8. Histogram showing the distribution of AGESM33 cloud velocities with respect to M33. The clouds we have described as 'discrete' are represented by the black edged bars and the clouds that show some low column density connection to M33 are represented by the blue bars.

identify one particularly interesting H I cloud AGESM33-31 that has many of the characteristics of $\mathrm{H}_{\mathrm{I}}$ distributed in the disc of a galaxy yet there is no known optical counterpart associated with it. This object has a total $\mathrm{H}_{\mathrm{I}}$ mass of $1.22 \times 10^{7} \mathrm{M}_{\odot}$ if at the distance of M33. However, we find that there are numerous other $\mathrm{H}$ I clouds in our observed region with similar velocities to AGESM33-31 these may be debris associated with the Magallanic stream. We are not able to rule out the Magellanic stream interpretation and the weight of evidence may actually support this conclusion.

\section{ACKNOWLEDGEMENTS}

This work is based on observations collected at Arecibo Observatory. The Arecibo Observatory is operated by SRI International under a cooperative agreement with the National Science Foundation (AST-1100968), and in alliance with Ana G. Méndez-Universidad Metropolitana, and the Universities Space Research Association.

RT is supported by the Tycho project LG14013, the project RVO:67985815 and by the Czech Science Foundation project P209/12/1795.

\section{REFERENCES}

Auld R. et al., 2006, MNRAS, 371, 1617

Barnes D. G. et al., 2001, MNRAS, 322, 486

Bekki K., 2008, MNRAS, 390, L24

Blitz L., Spergel D. N., Teuben P. J., Hartmann D., Burton W. B., 1999, ApJ, 514,818

Blitz L., Spergel D. N., Teuben P. J., Hartmann D., 2004, in van Woerden H., Wakker B. P., Schwarz U. J., de Boer K. S., eds, Astrophysics and Space Science Library, Vol. 312, High Velocity Clouds. Kluwer, Dordrecht, p. 297

Braun R., Burton W. B., 1999, A\&A, 341, 437

Braun R., Thilker D. A., 2004, A\&A, 417, 421 (BT04)

Burton W. B., Braun R., Chengalur J. N., 2001, A\&A, 369, 616

Chapman S. C. et al., 2013, MNRAS, 430, 37

Corbelli E., 2003, MNRAS, 342, 199

Cortese L. et al., 2008, MNRAS, 383, 1519

Davé R., Hernquist L., Katz N., Weinberg D. H., 1999, ApJ, 511, 521

Davé R., Spergel D. N., Steinhardt P. J., Wandelt B. D., 2001, ApJ, 547, 574

Davies J. I. et al., 2011, MNRAS, 415, 1883 
Freedman W. L., Wilson C. D., Madore B. F., 1991, ApJ, 372, 455

Grossi M., Giovanardi C., Corbelli E., Giovanelli R., Haynes M. P., Martin A. M., Saintonge A., Dowell J. D., 2008, A\&A, 487, 161 (G08)

Grossi M., Hwang N., Corbelli E., Giovanardi C., Okamoto S., Arimoto N., 2011, A\&A, 533, A91

Huxor A., Ferguson A. M. N., Barker M. K., Tanvir N. R., Irwin M. J., Chapman S. C., Ibata R., Lewis G., 2009, ApJ, 698, L77

Klypin A., Kravtsov A. V., Valenzuela O., Prada F., 1999, ApJ, 522, 82

McConnachie A. W., 2012, AJ, 144, 4

Martin N. F. et al., 2009, ApJ, 705, 758

Minchin R. F. et al., 2010, AJ, 140, 1093

Nidever D. L., Majewski S. R., Butler Burton W., Nigra L., 2010, ApJ, 723, 1618

Oey M. S., Groves B., Staveley-Smith L., Smith R. C., 2002, AJ, 123, 255

Putman M. E. et al., 2009, ApJ, 703, 1486

Sarajedini A., Barker M. K., Geisler D., Harding P., Schommer R., 2006, AJ, 132, 1361

Sault R. J., Teuben P. J., Wright M. C. H., 1995, in Shaw R. A., Payne H. E., Hayes J. J. E., eds, ASP Conf. Ser. Vol. 77, Astronomical Data Analysis Software and Systems IV. Astron. Soc. Pac., San Francisco, p. 433

Stadel J., Potter D., Moore B., Diemand J., Madau P., Zemp M., Kuhlen M., Quilis V., 2009, MNRAS, 398, L21

Sternberg A., McKee C. F., Wolfire M. G., 2002, ApJS, 143, 419

Taylor R., Davies J. I., Auld R., Minchin R. F., Smith R., 2013, MNRAS, 428, 459

Taylor R., Minchin R. F., Herbst H., Davies J. I., Rodriguez R., Vazquez C., 2014, MNRAS, 443, 2634 (AGES VII)
Thilker D. A., Braun R., Walterbos R. A. M., 2002, in Taylor A. R., Landecker T. L., Willis A. G., eds, ASP Conf. Ser. Vol. 276, Seeing Through the Dust: The Detection of HI and the Exploration of the ISM in Galaxies. Astron. Soc. Pac., San Francisco, p. 370

Wakker B. P., 1991, A\&A, 250, 499

Westmeier T., Braun R., Thilker D., 2005, A\&A, 436, 101

Wolfe S. A., Pisano D. J., Lockman F. J., McGaugh S. S., Shaya E. J., 2013, Nature, 497, 224

Wright M. C. H., 1979, ApJ, 233, 35

\section{SUPPORTING INFORMATION}

Additional Supporting Information may be found in the online version of this article:

Appendix A. Column density maps, velocity maps and spectra of the clouds around M33.

(http://www.mnras.oxfordjournals.org/lookup/suppl/doi:10.1093/ mnras/stv2684/-/DC1).

Please note: Oxford University Press is not responsible for the content or functionality of any supporting materials supplied by the authors. Any queries (other than missing material) should be directed to the corresponding author for the article.

This paper has been typeset from a $\mathrm{T}_{\mathrm{E}} \mathrm{X} / \mathrm{L} \mathrm{T}_{\mathrm{E}} \mathrm{X}$ file prepared by the author. 